\title{
GUEST EDITORIAL \\ Ketamine: translating mechanistic discoveries into the next generation of glutamate modulators for mood disorders
}

\author{
Molecular Psychiatry (2017) 22, 324-327; doi:10.1038/mp.2016.249; \\ published online 10 January 2017
}

Over the last decade, numerous controlled studies have consistently described rapid, robust, and relatively sustained antidepressant response rates associated with single-dose ketamine infusions. Indeed, antidepressant response rates at 4, 24, and $72 \mathrm{~h}$ were $50 \%, 70 \%$, and $35 \%$, respectively, in individuals with treatment-resistant depression (TRD) who received a single ketamine infusion. ${ }^{1,2}$ Furthermore, a recent meta-analysis of seven trials (encompassing 147 ketamine-treated patients) found that a single ketamine infusion produced rapid, yet transient, antidepressant effects, with odds ratios for response and transient remission of symptoms at $24 \mathrm{~h}$ equaling 9.87 (4.37-22.29) and 14.47 (2.67-78.49), respectively. ${ }^{3}$ It is important to note that, to date, no other treatment for depression has demonstrated comparable effects in terms of the magnitude of response to a single dose for TRD patients. Notably, ketamine has also been found to rapidly and robustly improve key dimensions such as anhedonia, fatigue, and suicidal thoughts in individuals with treatment-resistant major depressive disorder or bipolar depression. $^{4-7}$

Given these extremely promising findings, the past decade has been marked by an explosion of research seeking to understand the neurobiological underpinnings of rapid antidepressant response. ${ }^{8-11}$ Ketamine use in clinical settings has seen even more rapid growth, despite the fact that ketamine's off-label clinical use in the community is still unregulated and not standardized. ${ }^{12}$ Concomitantly, concerns surrounding chronic ketamine use and risk of neurotoxicity, cystitis, dissociative side effects, and abuse remain largely unresolved. To address such concerns, efforts are underway to bring ketamine to the market, standardize its use, and determine its real-world effectiveness (see, for instance, http://www.pcori.org/research-results/2016/electro convulsive-therapy-versus-ketamine-severe-resistant-depression). Here, we seek to summarize the ongoing evolution of theories regarding ketamine's mechanism of action; it is our hope that such information will be useful in determining which steps can best aid ongoing efforts to develop the next generation of antidepressant treatments that are safe, well-tolerated, and rapid-acting.

The original hypothesis that N-methyl-D-aspartate (NMDA) antagonists had antidepressant effects-which was based on a series of elegant preclinical studies ${ }^{13}$ - has been the prevailing theory to explain ketamine's antidepressant effects for the better part of almost three decades. Following a several years-long lull in the development of NMDA antagonists, the first report of ketamine's rapid antidepressant effects in a small group of depressed subjects appeared in $2000 .{ }^{14}$ Following a second yearslong lull, in 2006, a hypothesis-driven study replicated the original findings of rapid onset of antidepressant effects in a larger sample of TRD patients who received a single ketamine infusion. The latter study led to an exponential surge in preclinical and clinical studies examining ketamine use in mood disorders. ${ }^{1}$

At the cellular and molecular level, research has attempted to better characterize the neurobiological processes implicated in ketamine's rapid antidepressant effects following the initial NMDA blockade. This research has led to important insights in addition to identifying alternate targets for drug discovery with the goal of maintaining ketamine's favorable therapeutic profile but eliminating areas of concern associated with its use, such as dissociative side effects and abuse potential. ${ }^{15}$ Some of the alternate theories currently being investigated to explain ketamine's actions are explored in greater detail below.

The original preclinical evidence linking NMDA receptor antagonism and ketamine's rapid antidepressant effects led to a decade of preclinical and clinical trials with NMDA receptor antagonists. During this time, ketamine's antidepressant effects were consistently replicated in many pilot trials, and the onset and offset of antidepressant efficacy with a single administration became well characterized; ${ }^{1}$ such studies also helped characterize the side effects associated with ketamine and the time frame in which these were likely to occur. In addition, other studies exploring ketamine enantiomers have shown exciting clinical results using different dosing routes and frequency. However, efforts to develop a 'better' or alternate version of ketamine were fraught with difficulties from the start. Several broad and subunitselective NMDA antagonists that had been shelved after failing to demonstrate efficacy for other indications were reintroduced in the clinic as proof-of-concept agents for TRD. It is clear from these trials that some of these alternate ketamine NMDA receptor antagonists did indeed have some antidepressant properties. Nevertheless, none of them possessed all of ketamine's positive traits, including: (1) rapid onset of antidepressant effects, (2) robustness of efficacy, (3) sustained efficacy with a single administration, and (4) efficacy in TRD. In addition, no other NMDA antagonists appeared to possess the richness of ketamine's therapeutic effects including its antisuicidal properties, its antianhedonic effects, and its therapeutic potential in other disorders such as post-traumatic stress disorder or obsessive compulsive disorder.

Investigators wondered whether the modest efficacy seen with other NMDA receptor antagonists might be due to dose. That is, to produce few or no dissociative effects or elevations in blood pressure, doses of NMDA antagonists other than ketamine were purposely kept low and not increased, with the concomitant result that the drugs were better tolerated than ketamine but had limited efficacy. Whether these other drugs would have antidepressant effects and a side effect profile similar to ketamine's with higher doses remains unknown and, unfortunately, many of those experiments were not possible for a number of reasons. It should be noted that a number of NMDA receptor antagonists or glutamatergic NMDA receptor modulators are presently being studied that might conceivably yield promising results (for example, Rapastinel, CERC-301). ${ }^{1}$ However, none of the other NMDA antagonists tested to date have shown the same rapid, robust, and sustained antidepressant effects as ketamine; in other words, they are simply not ketamine.

Notably, convergent evidence from behavioral, cellular, and molecular ketamine studies supports the theory that enhanced aamino-3-hydroxy-5-methyl-4-isoxazolepropionic acid (AMPA) receptor activity-with a concomitant increase in synaptic plasticity-is critical to ketamine's mechanism of action and may be the key to developing similarly rapid-acting antidepressants. Within the tripartite glutamate synapse, ${ }^{16}$ AMPA receptors work in tandem with NMDA receptors. Although early NMDA receptor 
blockade initially increases synaptic glutamate release, this effect leads to preferential activation of AMPA receptor activity in critical neuronal circuits, particularly the cortico-limbic-striatal circuits. AMPA receptor trafficking also plays a key role in activating plasticity signaling cascades; relatedly, the delivery of AMPA receptors to the stimulated synapse-particularly postsynaptic AMPA-is key to synaptic strength. ${ }^{17}$

This AMPA throughput model was first proposed almost a decade ago in a study demonstrating that pre-treatment with an AMPA receptor antagonist blocked ketamine's antidepressant-like effects and also upregulated hippocampal phosphorylated GluR1 AMPA receptors. ${ }^{18}$ This model posits that AMPA's restorative effects result from a rapid increase in glutamate levels and the associated AMPA receptor insertion. Building on this work, Duman and colleagues found that direct blockade of NMDA receptors on inhibitory gamma aminobutyric acid (GABA)-ergic neurons in the prefrontal cortex led to a series of other important changes before AMPA activation, resulting in disinhibition of pyramidal neurons; this, in turn, led to increased glutamate release and enhanced signaling via its AMPA receptors. ${ }^{19}$

Because AMPA appeared critical to ketamine's antidepressant effects, researchers reasoned that AMPA positive modulatorsalso known as AMPAkines-could positively influence mood. Previous studies evaluating AMPAkines in mood disorders achieved mixed results, potentially due to low bioavailability and toxicity profile. ${ }^{20}$ To overcome the issue of low bioavailability, other AMPAkines were recently developed and tested in initial Phase 1 and 2 studies, particularly coluracetam $(\mathrm{BCl}-540)^{21}$ and ORG-26576. ${ }^{22}$ Both agents showed preliminary therapeutic effects in major depressive disorder when tested in small samples. ${ }^{23,24}$

Additional targets for ketamine's mechanism of action are also being explored; some of these postulate enhanced AMPA throughput. For instance, Rapastinel (Glyx-13), an NMDA receptor modulator with glycine-like properties, appears to have rapid antidepressant efficacy in treatment-resistant depression and, in preclinical studies, has been shown to activate AMPA receptors. ${ }^{25}$ 4-Cl-KYN (AV-101), a glycine receptor antagonist, also requires AMPA/kainate receptor activation, as highlighted by the ability of pre-treatment with an AMPA receptor antagonist to abolish their antidepressant-like effects in animal models. ${ }^{26}$ It should be noted that no evidence presently implicates AMPA receptor activation in conjunction with the NMDA receptor antagonist CERC-301 or the NMDA channel blocker AZD6765 (Lanicemine). ${ }^{27}$

It should also be noted that there are other ways to achieve increased AMPA throughput, though these have not yet been clinically tested. In addition to the direct effects of AMPA throughput, activation of the AMPA receptor targeting at mammalian target of rapamycin (mTOR), eukaryotic elongation factor (eEF2), and brain-derived neurotrophic factor (BDNF) signaling pathways may also be relevant to the rapid antidepressant effects of ketamine and related molecules. mTOR has multiple intracellular functions, including translation of proteins involved in synaptic plasticity and memory. Ketamine's ability to activate mTOR and multiple intracellular signaling cascades seems to be related to the increased AMPA receptor throughput and neuroproliferative effects in downstream targets regulated by ketamine. ${ }^{28}$ In this context, activation of the mTOR signaling pathway via AMPA receptors was found to be linked to ketamine's rapid antidepressant-like effects and thought to rapidly reverse stress- and/or depression-mediated deficits. Supporting a key role for mTOR in the molecular effects of ketamine, another study found that a single subanesthetic dose of ketamine rapidly upregulated mTOR signaling and potentiation of synaptic transmission mediated by AMPA receptors; ${ }^{19}$ however, this finding has not always been replicated across studies. ${ }^{29}$

With regard to the potential role of eEF2 and BDNF signaling in ketamine's rapid antidepressant effects, ketamine is known to increase BDNF signaling via post-synaptic AMPA receptor stimulation, permitting calcium influx and the resultant activity- dependent exocytosis of BDNF. It has also been hypothesized that ketamine suppresses eEF2 kinase activity that, in turn, reduces the suppression of protein translation, resulting in an upregulation of BDNF. ${ }^{30}$ Alternately, these modulatory effects seem to underlie ketamine's blockade at resting NMDA receptors, which has been shown to affect specific downstream plasticity targets, particularly eEF2 inhibition. ${ }^{30}$ Ketamine is also known to increase central and peripheral BDNF levels, which has been associated with changes in the number and function of synaptic connections in diverse preclinical studies. BDNF upregulation also induces acute changes in synaptic plasticity, mostly through increased surface expression of hippocampal AMPA receptors. ${ }^{31,32}$ These effects seem to be associated with the effects of AMPA activity on neuronal surface expression that consequently stimulate quick (30 min) eEF2- and BDNF-dependent potentiation. ${ }^{30,31}$

Glycogen synthase kinase 3 (GSK3) is another key target, and its inhibition has also been shown to be necessary for ketamine's rapid antidepressant-like effects mediated by AMPA receptors. ${ }^{21}$ One study found that mice expressing constitutively active GSK3 experienced no antidepressant-like effects in response to ketamine. ${ }^{33}$ More recently, the same group demonstrated that administering an antidepressant dose of ketamine increased cell membrane AMPA GluR1 levels by inhibiting GSK3, suggesting that GSK3 inhibition makes a mechanistic contribution to ketamine's rapid antidepressant action based on the AMPA throughput model. ${ }^{34}$ Furthermore, other glutamate receptors, monoamines, and G-protein-coupled receptors can also regulate AMPA receptors via protein phosphorylation and/or dephosphorylation mechanisms. ${ }^{23}$

Building on this work, investigators began exploring those cellular and molecular ketamine targets that are largely NMDA receptor-independent. Interestingly, recent preclinical studies evaluating ketamine and its metabolites support mechanisms of action beyond simple NMDA antagonism to explain the rapid and sustained antidepressant effects of these molecules, with a particular emphasis on enhanced AMPA to NMDA throughput (Figure 1). Of particular interest, a recent study found that ketamine metabolites activate AMPA receptors through an NMDAindependent mechanism at concentrations that equate to the Cmax of brain exposure after i.p. administration of antidepressant doses in mice. ${ }^{35}$ Ketamine is rapidly broken down into diverse metabolites that include the hydroxyketamines (HKs), dehydronorketamine, and hydroxynorketamine (HNK) (Figure 2). Dehydronorketamine and HNK are downstream metabolites of ketamine and norketamine, respectively. Norketamine's byproduct, $(2 R, 6 R)-\mathrm{HNK}$, is the major HNK metabolite found in the rodent brain as well as in human plasma after ketamine administration. It strongly activates hippocampal AMPA receptors and increases GluR1 chronically, but does not inhibit the NMDA receptor. ${ }^{35,36}$ As with ketamine, pre-treatment with the AMPA receptor antagonist NBQX prevented the acute antidepressant effects of $(2 R, 6 R)$-HNK injection. Furthermore, maintenance of $(2 R, 6 R)$-HNK's ongoing antidepressant-like effects were prevented with NBQX. These preclinical findings suggest that synaptic AMPA activation and related increased synaptic activity play a key role in the sustained antidepressant effects associated with ketamine metabolites. ${ }^{35}$ Intriguingly, these findings also echo and expand our decade-old original hypothesis, ${ }^{18,37,38}$ which posited that increased glutamatergic throughput of AMPA receptors relative to NMDA receptors was key to explaining ketamine's rapid antidepressant effects; this model was recently expanded to explain the sustained antidepressant effects of ketamine's HNK metabolite $((2 S, 6 S ; 2 R, 6 R)-H N K) .{ }^{35}$ However, to date, no clinical study has evaluated the potential rapid and sustained antidepressant effects of this ketamine metabolite in depressed patients, or confirmed that AMPA throughput is critical to ketamine's acute and sustained antidepressant effects in humans. Such studies are planned.

Finally, ketamine acts on several other targets beyond the glutamatergic system; these remain largely unexplored in the 


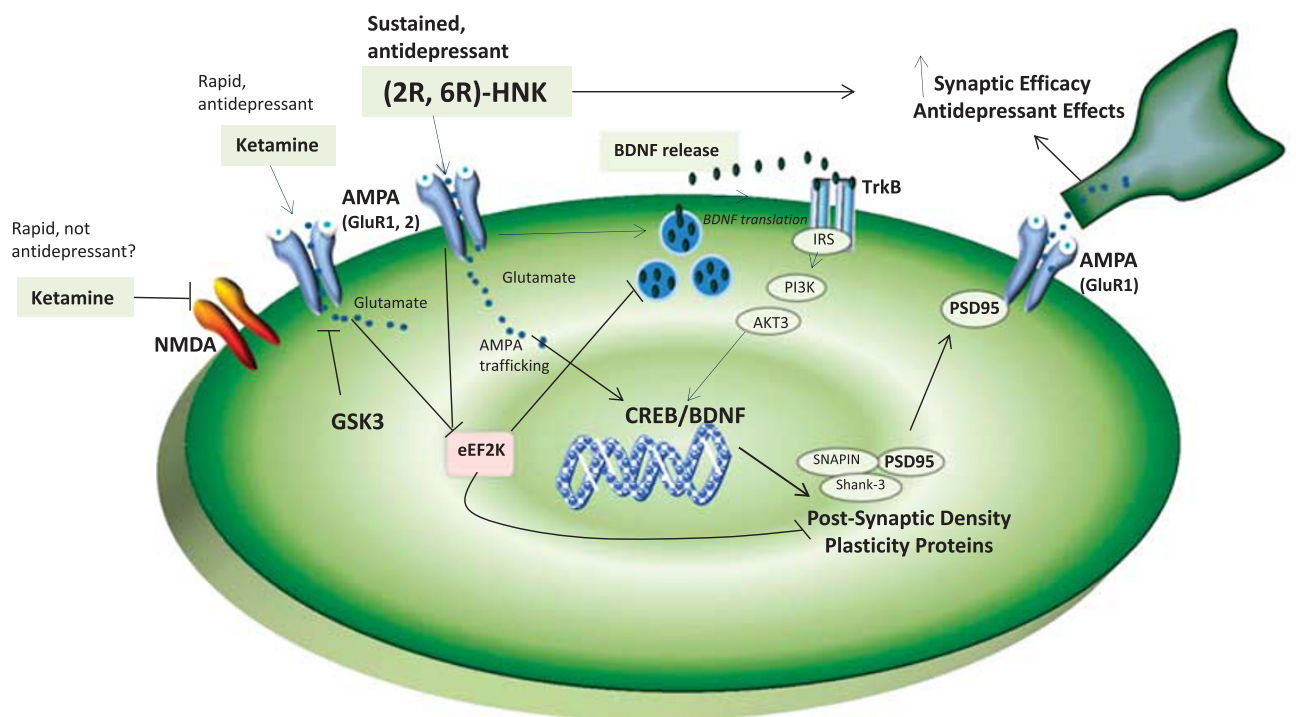

Figure 1. Enhanced AMPA to NMDA throughput as a key mechanism for the rapid and sustained antidepressant efficacy of ketamine and its metabolite $(2 R, 6 R)$-HNK. In this model, AMPA receptor activation (but not NMDA antagonism) is the key mechanism that induces antidepressant effects via activation and blockade of different downstream targets. Glutamate binds to AMPA receptors, favoring activation of intracellular AMPA trafficking and BDNF release. AMPA activation inhibits eEF2 kinase and increases BDNF synthesis, with a rapid peak in glutamate levels and activation of plasticity through BDNF/CREB transcription. AMPA trafficking induced by ketamine-and potentially by its metabolite $(2 R, 6 R)$-HNK - may activate CREB transcription and postsynaptic density proteins, particularly PSD95. PSD95 stabilizes AMPA receptors to the synapse and increases synaptic activity and synaptogenesis, thus inducing rapid and relatively sustained antidepressant effects. AMPA, $\alpha$-amino-3-hydroxy-5-methyl-4-isoxazolepropionic acid; BDNF, brain-derived neurotrophic factor; CREB, cyclic adenosine monophospate response element binding protein; eEF2, eukaryotic elongation factor; HNK, hydroxynorketamine; NMDA, N-methyl-Daspartate; PSD95, postsynaptic density protein 95.

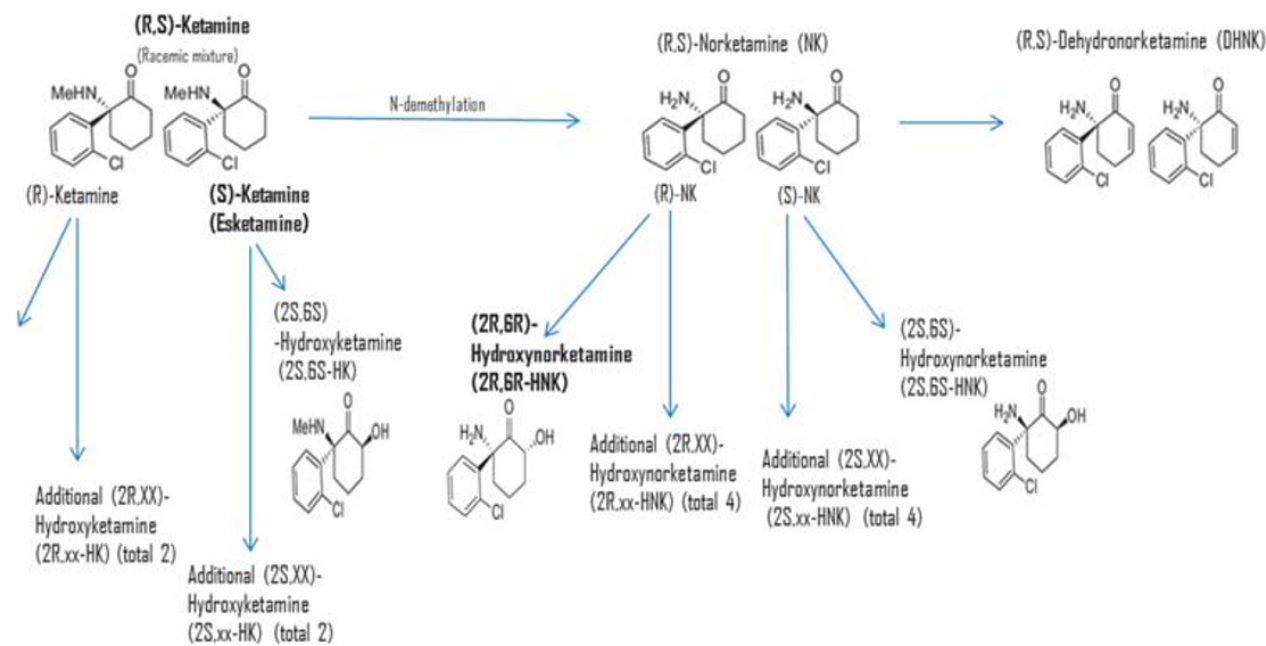

Figure 2. Ketamine metabolites: implications for therapeutics in mood disorders. $(R, S)$-ketamine is a racemic mixture containing equal amounts of optical isomers that have almost identical pharmacokinetic properties but different pharmacodynamic effects. Ketamine is metabolized via cytochrome P450 (CYP). CYP 3A4 is the main enzyme responsible for ketamine's N-demethylation to norketamine (NK). Norketamine is subsequently metabolized by multiple hydroxylation steps and is mostly dehydrogenated to yield (R,S)-dehydronorketamine (DHNK). It is next transformed into hydroxynorketamine (HNK) and finally excreted in bile and urine after glucuronoconjugation. (R,S)ketamine, (S)-ketamine, and the metabolite $(2 R, 6 R)$-HNK (in bold) have shown rapid, and in some cases sustained, antidepressant effects in clinical or preclinical studies.

context of its rapid antidepressant effects. For instance, ketamine induces aminergic activation and inhibits reuptake, and also targets delta opioid and mu-receptor function. ${ }^{23}$ Adipokines also appear to be affected by ketamine, which alters levels of leptin, resistin, and adiponectin; of the three, adiponectin levels were the strongest predictor of antidepressant response to ketamine, ${ }^{39}$ supporting ketamine's putative role in reversing metabolic dysfunction during mood episodes. All of these mechanisms are worthy of further exploration and underscore the potential complexity of the different mechanisms implicated in ketamine's antidepressant effects. 
In conclusion, when it comes to elucidating ketamine's underlying mechanisms of action, several promising areas of further study emerge. Pharmacological approaches that can bypass the initial neurobiological changes associated with NMDA receptor antagonism and its related psychotomimetic effects, as well as reverse dysregulation of critical neural circuits and cellular signaling at these pathways/targets (for example, ketamine metabolites), may be a promising avenue. To synthesize much of the evidence reviewed above, recent studies have reinforced that AMPA receptor activation is important to ketamine's rapid and sustained therapeutic effects, specifically through glutamate release and the consequent stimulation of downstream pathways; these late-phase effects include a key role for ketamine's metabolites targeting at eEF2 and the BDNF pathway. This restorative effect results from elevated glutamate levels (glutamate surge-although not all investigations support the glutamate surge hypothesis) and increased AMPA receptor insertion (together with an increase in amines) as well as increased prefrontal cortex synaptogenesis and connectivity. These alternative mechanisms of action would be expected to trigger longterm changes in synaptic activation related to sustained antidepressant actions, but occur independently of the initial NMDA receptor blockade (Figure 1). Overall, NMDA receptorindependent mechanisms are comparatively underexplored but particularly appealing for the development of new agents capable of inducing sustained antidepressant effects; most importantly, this alternative path to discovery may include the development of an improved version of ketamine by exploring ketamine's metabolites, which are expected to bypass its dissociative adverse effects, as gleaned from studies with $(2 R, 6 R)$-HNK.

Given the complexity of the material reviewed above, we note that it is unlikely that a single mechanism could be responsible for ketamine's unique antidepressant effects. Ongoing studies examining the therapeutic profile of ketamine's metabolites and its downstream targets, as well as other ways of enhancing AMPA throughput mechanisms, should prove particularly insightful. Furthermore, the precise sequence of the cellular and molecular changes implicated in ketamine's mechanism of action remain to be identified.

\section{CONFLICT OF INTEREST}

Dr Zarate Jr is listed as co-inventor on a patent application for the use of $(2 R, 6 R)$ hydroxynorketamine and $(2 S, 6 S)$-hydroxynorketamine in the treatment of depression, anxiety, anhedonia, suicidal ideation, and post-traumatic stress disorders; he has assigned his patent rights to the U.S. government but will share a percentage of any royalties that may be received by the government. Dr Machado-Vieira has no conflict of interest to disclose, financial or otherwise.

\section{ACKNOWLEDGMENTS}

Funding for this work was supported by the Intramural Research Program at the National Institute of Mental Health, National Institutes of Health (IRP-NIMH-NIH; ZIA $\mathrm{MH002857),} \mathrm{by} \mathrm{a} \mathrm{NARSAD} \mathrm{Independent} \mathrm{Investigator} \mathrm{Award} \mathrm{to} \mathrm{Dr} \mathrm{Zarate} \mathrm{Jr,} \mathrm{and} \mathrm{by} \mathrm{a}$ Brain and Behavior Mood Disorders Research Award to Dr Zarate Jr. We thank the 7SE research unit and staff for their support.

CA Zarate Jr and R Machado-Vieira Experimental Therapeutics and Pathophysiology Branch, Intramural Research Program, National Institute of Mental Health, National Institutes of Health, Bethesda, MD, USA E-mail: zaratec@mail.nih.gov

\section{REFERENCES}

1 ladarola ND, Niciu MJ, Richards EM, Vande Voort JL, Ballard ED, Lundin NB et al. Ther Adv Chronic Dis 2015; 6: 97-114.

2 Zarate CA Jr., Singh JB, Carlson PJ, Brutsche NE, Ameli R, Luckenbaugh DA et al. Arch Gen Psychiatry 2006; 63: 856-864.

3 Newport DJ, Carpenter LL, McDonald WM, Potash JB, Tohen M, Nemeroff CB et al. Am J Psychiatry 2015; 172: 950-966.

4 Diazgranados N, Ibrahim L, Brutsche N, Newberg A, Kronstein P, Khalife S et al. Arch Gen Psychiatry 2010; 67: 793-802.

5 Lally N, Nugent AC, Luckenbaugh D, Niciu MJ, Roiser JP, Zarate CA. J Psychopharmacol 2015; 29: 596-607.

6 Lally N, Nugent AC, Luckenbaugh DA, Ameli R, Roiser JP, Zarate CA. Transl Psychiatry 2014; 4: e469.

7 Saligan LN, Luckenbaugh D, Slonena EE, Machado-Vieira R, Zarate CAJ. J Affect Disord 2016; 194: 115-119.

8 Abdallah CG, Sanacora G, Duman RS, Krystal JH. Annu Rev Med 2015; 66: 509-523.

9 Niciu MJ, Mathews DC, Nugent AC, lonescu DF, Furey ML, Richards EM et al. Depress Anxiety 2014; 31: 297-307.

10 Gerhard DM, Wohleb ES, Duman RS. Drug Discov Today 2016; 21: 454-464.

11 Lener MS, Niciu MJ, Ballard ED, Park M, Park LT, Nugent AC et al. Biol Psychiatry 2016 (e-pub ahead of print 12 May).

12 Sanacora G, Heimer H, Hartman D, Mathew SJ, Frye M, Nemeroff CB et al. Neuropsychopharmacology 2016 (e-pub ahead of print 12 October).

13 Skolnick P, Popik P, Trullas R. Trends Pharmacol Sci 2009; 30: 563-569.

14 Berman RM, Cappiello A, Anand A, Oren DA, Heninger GR, Charney DS et al. Biol Psychiatry 2000; 47: 351-354.

15 Machado-Vieira R, Salvadore G, Diazgranados N, Zarate CAJ. Pharmacol Ther 2009; 123: $143-150$.

16 Machado-Vieira R, Manji HK, Zarate CA. Neuroscientist 2009; 15: 525-539.

17 Malinow R, Malenka RC. Annu Rev Neurosci 2002; 25: 103-126.

18 Maeng S, Zarate CA, Du J, Schloesser RJ, McCammon J, Chen G et al. Biol Psychiatry 2008; 63: 349-352.

19 Li N, Lee B, Liu R-J, Banasr M, Dwyer JM, Iwata M et al. Science 2010; 329: 959-964.

20 Menniti FS, Lindsley CW, Conn PJ, Pandit J, Zagouras P, Volkmann RA. Curr Top Med Chem 2013; 13: 26-54.

21 Dutta A, McKie S, Deakin JF. Psychiatry Res 2015; 225: 1-13.

22 Nations KR, Dogterom P, Bursi R, Schipper J, Greenwald S, Zraket D et al. J Psychopharmacol 2012; 26: 1525-1539.

23 Machado-Vieira R, Henter ID, Zarate CA. Prog Neurobiol 2015 (e-pub ahead of print 23 December); pii: S0301-0082(15)30038-1; doi: 10.1016/j.pneurobio.2015.12.001.

24 Martinowich K, Jimenez DV, Zarate CA, Manji HK. Mol Psychiatry 2013; 18: 856-863.

25 Yang B, Zhang JC, Han M, Yao W, Yang C, Ren Q et al. Psychopharmacol (Berl) 2016; 233: 3647-3657.

26 Burgdorf J, Zhang XL, Nicholson KL, Balster RL, Leander JD, Stanton PK et al. Neuropsychopharmacology 2013; 38: 729-742.

27 Jaso BA, Niciu MJ, ladarola ND, Lally N, Richards EM, Park M et al. Curr Neuropharmacol 2016 (e-pub ahead of print 21 March).

28 Chen K-T, Tsai M-H, Wu C-H, Jou M-J, Wei I-H, Huang C-C. Front Behav Neurosci 2015; 9: 162.

29 Popp S, Behl B, Joshi JJ, Lanz TA, Spedding M, Schenker E et al. F1000Research 2016; 5: 634.

30 Autry AE, Adachi M, Nosyreva E, Na ES, Los MF, Cheng PF et al. Nature 2011; 475: 91-95.

31 Nosyreva E, Szabla K, Autry AE, Ryazanov AG, Monteggia LM, Kavalali ET. J Neurosci 2013; 33: 6990-7002.

32 Kavalali ET, Monteggia LM. Am J Psychiatry 2012; 169: 1150-1156.

33 Beurel E, Song L, Jope R. Mol Psychiatry 2011; 16: 1068-1070.

34 Beurel E, Grieco SF, Amadei C, Downey K, Jope RS. Bipolar Disord 2016; 18: $473-480$.

35 Zanos P, Moaddel R, Morris PJ, Georgiou P, Fischell J, Elmer Gl et al. Nature 2016; 533: $481-486$

36 Moaddel R, Abdrakhmanova G, Kozak J, Jozwiak K, Toll L, Jimenez L et al. Eur J Pharmacol 2013; 698: 228-234.

37 Du J, Machado-Vieira R, Maeng S, Martinowitch K, Manji HK, Zarate CA Jr. Drug Discov Today Ther Strateg 2006; 3: 519-526.

38 Maeng S, Zarate CA. Curr Psychiatry Rep 2007; 9: 467-474.

39 Machado-Vieira R, Gold PW, Luckenbaugh D, Ballard ED, Richards EM, Henter ID et al. Mol Psychiatry 2016 (this issue). 